\title{
Tyrphostin B42 attenuates trichostatin A-mediated resistance in pancreatic cancer cells by antagonizing IL-6/JAK2/STAT3 signaling
}

\author{
XING ZHANG $^{1 *}$, HONG LU $^{2 *}$, WEILONG HONG ${ }^{1}$, LEPING LIU $^{1}$, SILU WANG $^{1}$, \\ MENGTAO ZHOU ${ }^{1,3}$, BICHENG CHEN $^{1}$ and YONGHENG BAI ${ }^{1}$ \\ ${ }^{1}$ Key Laboratory of Diagnosis and Treatment of Severe Hepato-Pancreatic Diseases of Zhejiang Province, \\ Departments of ${ }^{2}$ Laboratory Medicine and ${ }^{3}$ Hepatobiliary Surgery, The First Affiliated Hospital \\ of Wenzhou Medical University, Wenzhou, Zhejiang 325000, P.R. China
}

Received July 1, 2017; Accepted January 24, 2018

DOI: $10.3892 / o r .2018 .6241$

\begin{abstract}
Drug-resistance is the key reason for the ineffectiveness of chemotherapy in pancreatic cancer. Thus, it is very important to explore the molecular mechanisms of drug-resistance and the methods of effective intervention. In the present study, we investigated the effect of tyrphostin B42, also called AG490, on histone deacetylase (HDAC) inhibitor trichostatin A (TSA)-induced resistance in pancreatic cancer cells (PCCs). Evidence from phase contrast microscope revealed that TSA-resistant cells (PANC-1-TSA) had higher proliferative activity than non-resistant cells (PANC-1). This over-proliferative activity induced by TSA may be associated with abnormal activation of JAK2/STAT3 signaling, which can be strengthened by interleukin-6 (IL-6), a STAT3-upstream inducer, resulting in enhanced expression of STAT3-downstream target genes including c-Myc, c-Src, HIF-1 $\alpha$, and CCND1. In addition, increased expression of Bcl-2 mRNA and decreased expression of Bax mRNA in PANC-1-TSA cells indicated that TSA induced the inhibition of mitochondrial-dependent
\end{abstract}

Correspondence to: Dr Yongheng Bai or Dr Bicheng Chen, Key Laboratory of Diagnosis and Treatment of Severe Hepato-Pancreatic Diseases of Zhejiang Province, The First Affiliated Hospital of Wenzhou Medical University, No. 2 Fuxue Lane, Wenzhou, Zhejiang 325000, P.R. China

E-mail: baiyongheng@hotmail.com

E-mail: bisonch@163.com

*Contributed equally

Abbreviations: FITC, fluorescein isothiocyanate; HDAC, histone deacetylase; JAK, Janus kinase; PCCs, pancreatic cancer cells; RT-qPCR, reverse-transcriptase quantitative polymerase chain reaction; STAT, signal transducers and activators of transcription; TSA, trichostatin A

Key words: tyrphostin B42 (AG490), pancreatic cancer, trichostatin A, chemotherapy resistance, IL-6/JAK2/STAT3 signaling apoptosis in PCCs. Tyrphostin B42 treatment evidently antagonized the activation of IL-6/JAK2/STAT3 in a dose-dependent manner. As a result, tyrphostin B42 inhibited the over-proliferative activity of PANC-1-TSA cells, and downregulated the expression of IL-6/JAK2/STAT3-downstream target genes. Moreover, tyrphostin B42 induced the apoptosis of PCCs by regulating the expression of mitochondrial-related genes. Therefore, these findings demonstrated that tyrphostin B42 attenuated TSA-mediated resistance in PCCs by antagonizing the IL-6/JAK2/STAT3 signaling.

\section{Introduction}

Pancreatic cancer is a type of aggressive malignant tumor with drug-resistance, poor prognosis and high mortality. The etiology of pancreatic cancer remains unclear, and the factors including genetics, diet and chronic pancreatitis contribute to the occurrence and development of pancreatic cancer. Due to the changes in lifestyle habits, dietary structure and an increase in environmental pollution, the incidence of pancreatic cancer has increased gradually $(1,2)$. Clinically, surgery is regarded as the main treatment method for pancreatic cancer. However, only a small number of patients with pancreatic cancer can undergo surgery. Patients who are unable to undergo surgery presently are treated with 5-fluorouracil, cisplatin, gemcitabine, or a combination of radiation therapy and chemotherapy (3-5). These auxiliary treatments can to some extent inhibit the further deterioration of patients with pancreatic cancer, but whether they can extend the life of patients warrants further clinical observations. Thus, it is urgent to develop new treatments and effective drugs for pancreatic cancer.

In recent years, the importance of epigenetic alterations has been confirmed in cancer development, including the role of aberrant DNA methylation and histone acetylation on aberrant silencing of multiple tumor-suppressor genes in a diversity of human cancers (6). HDAC inhibitors, which interfere with the function of histone deacetylase (HDAC), are emerging as potent anticancer agents as a result of their effective anti-proliferative activity in a wide variety of tumors, mediated by mitotic defects through the aberrant acetylation of histone 
and non-histone proteins (3,7). However, our previous study revealed that pancreatic cancer cells (PCCs) were resistant to HDAC inhibitor trichostatin A (TSA) (8). Over-proliferative activity and inhibition of apoptosis indicated that some proliferation-related signaling pathways may be involved in the process of TSA-mediated resistance in PCCs $(8,9)$.

According to previous studies, IL-6 is a well-studied proinflammatory cytokine with numerous links to a variety of malignancies (10). IL-6 has been noted to be overexpressed in patients with pancreatic cancer (11). In vivo, IL-6 can induce the phosphorylation of Janus kinase (JAK) via the activation of cytokine receptors, which then creates receptor docking sites for recruitment of cytoplasmic signal transducers and activators of transcription-3 (STAT) proteins. Once in the nucleus, STAT molecules bind specific promoter DNA sequences that result in the transcription of genes that regulate the proliferation, differentiation, and apoptosis of cancer cells (12). STAT3 belongs to a family of transcription factors that relay cytokine receptor generated signals into the nucleus $(13,14)$. Once in the nucleus, STAT molecules bind specific promoter DNA sequences that result in the transcription of genes that regulate cell proliferation, differentiation, and apoptosis (e.g., Bcl-2, CCND1 and c-Myc) $(11,12,15)$. The apoptosis-associated proteins Bcl-2 and Bax play an important role in regulating cell survival and are key transcriptional targets of STAT3 (16-18). Evidence has revealed that hypoxia-inducible factor $1 \alpha$ (HIF-1 $\alpha$ ) plays pivotal roles in tumor invasion, metastasis, and drug resistance. It has been well documented that hypoxia is one of the fundamental biological phenomena, which is related to the development and aggressiveness of pancreatic cancer $(19,20)$. Furthermore, inhibition of the STAT3/cyclinD1 pathway increased the sensitivity of cancer cells to chemotherapy (21).

In the present study, we first investigated the activity of IL-6/JAK2/STAT3 signaling in patients with pancreatic cancer and in PCCs with (PANC-1-TSA) or without (PANC-1) TSA resistance. Secondly, tyrphostin $\mathrm{B} 42$ had been reported to possess inhibitory activity on the proliferation of various tumor cells $(22,23)$, and to be associated with IL-6/JAK2/STAT3 signaling (24). Therefore, in the present study the effects of tyrphostin B42 on TSA-induced over-proliferation and resistance of PCCs were also evaluated. Our results revealed the crucial role of IL-6/JAK2/STAT3 signaling in TSA-resistance of PCCs, resulting in the inhibition of apoptosis and induction of proliferation. Treatment with tyrphostin B42 attenuated TSA-mediated resistance by antagonizing IL-6/JAK2/STAT3 signaling.

\section{Materials and methods}

Patients with pancreatic cancer. In the present study, a total of 96 patients with pancreatic cancer were enrolled from the First Affiliated Hospital of Wenzhou Medical University (Wenzhou, China) between March 2015 and August 2016. All pancreatic cancer cases, which were confirmed by pathological examination, enrolled in the current study had not received any radiation or chemotherapy before surgery. The demographic and clinical characteristics of the subjects are shown in Table I, including age, sex, smoking, tumor stage, TNM classification, lymph node metastasis, and vascular infiltration. Based on the TNM classification system promulgated by the American Joint Committee on Cancer (AJCC), the pathologic stage was divided into localized and aggressive cancer, as determined by transrectal ultrasound, magnetic resonance imaging (MRI) and emission computed tomography (ECT) (25).

All subjects were informed about the contents of the study and provided their informed consent. This study was approved by the Ethics Committee of Wenzhou Medical University.

Immunohistochemical analysis. Sections, 4- $\mu$ m-thick, from pancreatic cancer tissues in patients were dewaxed with xylene and hydrated using sequential ethanol $(100,95,85$, and $75 \%)$ and distilled water washes. The endogenous peroxidase was blocked with $3 \%$ hydrogen peroxide. Antigen retrieval was performed by heating the sections in $0.1 \%$ sodium citrate buffer $(\mathrm{pH}$ 6.0). The expression of IL-6 (CAS No: ab6672, 1:400; Abcam, Cambridge, MA, USA) was determined using the immunochemical streptavidin-peroxidase method. All samples were semi-quantitatively or quantitatively assessed by two independent investigators in a blinded manner. Slides were examined and images were captured using a DM4000 B LED microscope system (Leica Microsystems GmbH, Hannheim, Germany) and a DFC 420 C $5 \mathrm{M}$ digital microscope camera (Leica Microsystems $\mathrm{GmbH}$ ). For H-score assessment, 10 fields were chosen at random at an x400 magnification and the staining intensity in the malignant cell nuclei was scored as $0,1,2$, or 3 corresponding to the presence of negative, weak, intermediate, and strong brown staining, respectively. The total number of cells in each field and the number of cells stained at each intensity were counted. The average percentage of positive cells was calculated and the following formula was applied:

IHA $\mathrm{H}$-score $=(\%$ of cells stained at intensity category $1 \mathrm{x} 1)+(\%$ of cells stained at intensity category $2 \times 2)+(\%$ of cells stained at intensity category $3 \times 3)(26)$.

Cell culture and drug treatment. Human PCCs (PANC-1) were obtained from the American Type Culture Collection (ATCC ${ }^{\circledR}$ CRL-1469 ${ }^{\mathrm{TM}}$; Manassas, VA, USA). Trichostatin A (TSA; CAS No: 58880-19-6; Selleckchem, Houston, TX, USA)-induced resistance of PCCs (PANC-1-TSA) were generated in our laboratory. PANC-1 and PANC-1-TSA cells were maintained in DMEM (Gibco; Invitrogen; Thermo Fisher Scientific, Inc., Grand Island, NY,USA) and supplemented with $10 \%$ fetal bovine serum (FBS), $100 \mathrm{U} / \mathrm{ml}$ penicillin and $100 \mu \mathrm{g} / \mathrm{ml}$ streptomycin (all from Invitrogen; Thermo Fisher Scientific, Inc.) were treated with 5\% tyrphostin B42 (CAS No: 133550-30-8; Selleckchem) or recombinant IL-6 (CAS No: 200-06; PeproTech, Inc., Rocky Hill, NJ, USA) for $24 \mathrm{~h}$. Tyrphostin B42a and IL-6 were dissolved in $100 \%$ dimethyl sulfoxide (DMSO; Sigma-Aldrich, St. Louis, MO, USA) and diluted with the culture medium for experiments. The final concentration of DMSO for all treatments was maintained at $0.1 \%$.

Establishment of TSA-resistant PCCs (PANC-1-TSA). PANC-1-TSA was obtained by culture of PCCs (PANC-1) in vitro with intermittent increase of the concentration of TSA in the culture for 30 weeks. After cultivating PANC-1 cells with different concentrations of TSA for 1 week, we ascertained the cell death conditions and chose the concen- 
Table I. Demographic and characteristics of patients with pancreatic cancer.

\begin{tabular}{|c|c|c|c|c|c|}
\hline \multirow[b]{2}{*}{ Characteristics } & \multirow[b]{2}{*}{ Number (\%) } & \multicolumn{2}{|c|}{ IL-6 expression } & \multirow[b]{2}{*}{ P-value } & \multirow[b]{2}{*}{ Odds ratio $(95 \% \mathrm{CI})$} \\
\hline & & Negative (\%) & Positive (\%) & & \\
\hline Total & & 96 & $19(19.8)$ & $77(80.2)$ & \\
\hline \multicolumn{6}{|l|}{ Sex } \\
\hline Female & $25(26)$ & $5 \quad(5.2)$ & $20(20.8)$ & 0.976 & $0.982(0.314-3.075)$ \\
\hline Male & $71(74)$ & $14(14.6)$ & $57(59.3)$ & & \\
\hline \multicolumn{6}{|l|}{ Smoking } \\
\hline Yes & $44(45.8)$ & $9 \quad(9.4)$ & $35(36.5)$ & 0.881 & $0.926(0.339-2.532)$ \\
\hline No & $52(54.2)$ & $10(10.4)$ & $42(43.8)$ & & \\
\hline \multicolumn{6}{|l|}{ Age (years) } \\
\hline$\leq 60$ & $43(44.8)$ & $8 \quad(8.3)$ & 35 (39.6) & 0.793 & $1.146(0.415-3.162)$ \\
\hline$>60$ & $53(55.2)$ & $11(11.5)$ & $42(43.8)$ & & \\
\hline \multicolumn{6}{|l|}{ Tumor stage } \\
\hline $\mathrm{T} 1+\mathrm{T} 2$ & $8(8.3)$ & $3(3.1)$ & $5 \quad(5.2)$ & 0.197 & $2.100(0.446-9.891)^{\mathrm{a}}$ \\
\hline $\mathrm{T} 3+\mathrm{T} 4$ & $63(65.6)$ & $14(14.6)$ & $49(51.0)$ & & \\
\hline Unknown & $25(26.0)$ & $2(2.1)$ & $23(24.0)$ & & \\
\hline \multicolumn{6}{|c|}{ Lymph node metastasis } \\
\hline Yes & $38(39.6)$ & $3(3.1)$ & $35(36.5)$ & 0.027 & $4.321(1.082-17.252)^{\mathrm{b}}$ \\
\hline No & 37 (38.5) & $10(10.4)$ & $27(28.1)$ & & \\
\hline Unknown & $21(21.9)$ & $4 \quad(4.2)$ & $17(17.7)$ & & \\
\hline \multicolumn{6}{|c|}{ Tumor differentiation } \\
\hline Mildly & $19(19.8)$ & $9 \quad(9.4)$ & $10(10.4)$ & 0.003 & $6.120(1.999-18.734)^{\mathrm{c}}$ \\
\hline Moderately & $50(52.1)$ & $7 \quad(7.3)$ & $43(44.8)$ & & \\
\hline Poorly & $27(28.1)$ & $3(3.1)$ & $25(26.0)$ & & \\
\hline \multicolumn{6}{|l|}{ Vascular invasion } \\
\hline Perineural & $26(16.7)$ & $2(2.1)$ & $24(25.0)$ & 0.049 & $4.653(1.001-21.633)^{\mathrm{d}}$ \\
\hline Adipose tissue & $68(51.0)$ & $19(19.9)$ & $49(51.0)$ & & \\
\hline
\end{tabular}

${ }^{\mathrm{a}} \mathrm{P}=0.197,(\mathrm{~T} 3+\mathrm{T} 4)$ vs. $(\mathrm{T} 1+\mathrm{T} 2) ;{ }^{\mathrm{b}} \mathrm{P}=0.027$, metastasis vs. non-metastasis; ${ }^{\mathrm{c}} \mathrm{P}=0.003$, (poorly + moderately) vs. (mildly); ${ }^{\mathrm{d}} \mathrm{P}=0.035$, perineural vs. adipose tissue.

tration $(0.05 \mu \mathrm{mol} / \mathrm{l})$ of median lethal dose $\left(\mathrm{LD}_{80}\right)$ as the initial concentration to cultivate the resistant cell line. When cells grew stably and entered the logarithmic growth phase, the concentration gradually increased at $0.01 \mu \mathrm{mol} / 1$ every 2 weeks. After 15 concentration gradients and 30 weeks of cultivation, the final concentration was stable at $0.2 \mu \mathrm{mol} / 1$.

$C C K-8$ assay. Cell suspension (100 $\mu \mathrm{l} ; 5.0 \times 10^{3}$ cells/well) was dispensed in a 96-well plate with the outer wells left empty for the addition of water. The plate was pre-incubated for $24 \mathrm{~h}$ in humidified tyrphostin B42 and DMSO was used as a control. A CCK-8 solution $(10 \mu \mathrm{l})$ was added (Dojindo Molecular Technologies, Inc., Tokyo, Japan) to each well of the plate. The plate was then incubated for $4 \mathrm{~h}$. The absorbance at $450 \mathrm{~nm}$ was measured using a microplate reader (Thermo Fisher Scientific, Inc.). Six parallel wells were set up in each experiment, and all of the measurements were repeated at least three times.

Quantitative real-time polymerase chain reaction ( $q R T-P C R)$ analysis. Total RNA was isolated using TRIzol (Invitrogen;
Thermo Fisher Scientific, Inc.). The concentration and purity of RNA were determined using a spectrophotometer. cDNA was synthesized with reverse transcriptase (Thermo Fisher Scientific, Inc.). qRT-PCR assays were carried out using FastStart Universal SYBR Green Master Mix (Roche Diagnostics GmbH, Mannheim, Germany). PCR parameters were as follows: $95^{\circ} \mathrm{C}$ for $10 \mathrm{~min}$, then $95^{\circ} \mathrm{C}$ for $15 \mathrm{sec}$, and $60^{\circ} \mathrm{C}$ for $30 \mathrm{sec}$ for 40 cycles. The PCR assays were performed in triplicate and were conducted using a Real-Time PCR Detection System (ABI 7500; Applied Biosystems; Thermo Fisher Scientific, Inc., Carlsbad, CA, USA). The expression of mRNA was calculated using the $2^{-\Delta \Delta C t}$ method as previously described (27). Three replicate reactions per sample and endogenous control were used to ensure statistical significance. All the primers that were used in this study are listed in Table II.

Western blot analysis. Whole-cell protein extracts and nuclear protein extracts from PCCs were prepared with RIPA Lysis Buffer [50 mM Tris-HCl, pH 8.0, with 150 mM sodium chloride, 
Table II. qRT-PCR primers in this study.

\begin{tabular}{lll}
\hline Gene & \multicolumn{1}{c}{ Forward $\left(5^{\prime} \rightarrow 3^{\prime}\right)$} & \multicolumn{1}{c}{ Reverse $\left(5^{\prime} \rightarrow 3^{\prime}\right)$} \\
\hline HIF-1 $\alpha$ & GCAGCAACGACACAGAAACT & AGCGGTGGGTAATGGAGAC \\
CCND1 & CCTGTCCTACTACCGCCTCA & TCCTCCTCTTCCTCCTCCTC \\
c-Src & CGAGAAAGTGAGACCACGAA & TGCGGGAGGTGATGTAGAA \\
c-Myc & CCTCCACTCGGAAGGACTATC & TTCGCCTCTTGACATTCTCC \\
BCL-2 & CAACACAGACCCACCCAGA & TGGCTTCATACCACAGGTTTC \\
Bax & TTTCTGACGGCAACTTCAACTG & CGGAGGAAGTCCAATGTCCAG \\
GAPDH & TCCCATCACCATCTTCCAGG & GATGACCCTTTTGGCTCCC \\
\hline
\end{tabular}

$1.0 \%$ Igepal CA-630 [(NP-40]), $0.5 \%$ sodium deoxycholate, and $0.1 \%$ sodium dodecyl sulfate]) and a Nuclear Extract kit (Active Motif, Carlsbad, CA, USA), respectively, according to the manufacturer's instructions. Protein concentrations were determined using an assay kit (Bio-Rad Laboratories, Inc., Hercules, CA, USA). Lysates containing $100 \mu \mathrm{g}$ of protein were mixed with loading buffer containing 5\% $\beta$-mercaptoethanol and heated for $5 \mathrm{~min}$ at $100^{\circ} \mathrm{C}$. The samples were separated by sodium dodecyl sulfate-polyacrylamide gel electrophoresis and transferred onto nitrocellulose membranes by blotting. The membranes were probed with antibodies JAK2 (D2E12) (1:1,000; rabbit monoclonal; cat. no. 3230), STAT3 (124H6) (1:1,000; mouse monoclonal; cat. no. 9139), and p-STAT3 (D3A7) (1:1,000; rabbit monoclonal; cat. no. 9145; all from Cell Signaling Technolgy, Inc., Danvers, MA, USA) using an enhanced chemiluminescence kit (cat. no. 34095; Thermo Fisher Scientific), and GAPDH (1:5,000; cat. no. AP0063; Bioworld Technology, St. Louis Park, MN, USA) was used as a control.

Statistical analysis. All in vitro experiments were repeated at least three times to confirm the results. All the statistical calculations were performed using Prism 6.0 (GraphPad Software, Inc., San Diego, CA, USA) and the data were expressed as the mean \pm SEM. Statistical significance was determined with the Student's t-test (two-tailed) when comparing two groups of data set. P-values $<0.05$ were considered to indicate statistical significance. Asterisks shown in the figures indicate significant differences of the experimental groups in comparison with the corresponding control groups.

\section{Results}

IL-6/JAK2/STAT3 signaling is activated in pancreatic cancer and exacerbated by TSA. We first evaluated the activity of IL-6, an upstream inducer of JAK2/STAT3 signaling, in patients with pancreatic cancer. As shown in Fig. 1A and Table I, the expression levels of IL- 6 in pancreatic cancer tissues were increased, and positively associated with lymph node metastasis $(\mathrm{P}=0.027)$, tumor differentiation $(\mathrm{P}=0.003)$ and vascular infiltration $(\mathrm{P}=0.049)$ of pancreatic cancer. In pancreatic cancer patients, enhanced IL-6 expression had a high risk of lymph node metastasis [Odds ratio $(\mathrm{OR})=4.321$; 95\% CI: 1.082-17.252; $\mathrm{P}=0.027$; metastasis vs. non-metastasis), poorly differentiated $(\mathrm{OR}=6.120 ; 95 \% \mathrm{CI}: 1.999-18.734$; $\mathrm{P}=0.003$; (poorly + moderately) vs. mildly)], and perineural vascular infiltration $(\mathrm{OR}=4.653,95 \% \mathrm{CI}: 1.001-21.633$; $\mathrm{P}=0.035$; perineural vs. adipose), suggesting that IL-6 levels and downstream JAK2/STAT3 signaling may be essential for the development of pancreatic cancer.

We next investigated the activity of IL-6/JAK2/STAT3 signaling in different PCC cell lines. We found overexpression of JAK2 and STAT3, but not the phosphorylation of STAT3, in PCC cell lines including ASPC-1, SW1990, Puta 8988, and PANC-1 (Fig. 1B and C). Among them, PANC-1 cells exhibited the highest expression of the STAT3 protein. In addition, in PANC-1 cells, IL-6 activated JAK2/STAT3 signaling by inducing the expression and phosphorylation of STAT3 (Fig. 1D-F). Similarly, in PANC-1-TSA cells, IL-6 also induced the phosphorylation of STAT3, although the changes of STAT3 expression did not exhibit significance (Fig. 1D-F). These results identified again the crucial role of IL-6/JAK2/STAT3 signaling in pancreatic cancer. Notably, the activity of IL-6/JAK2/STAT3 signaling in PANC-1-TSA cells was higher when compared with those in PANC-1 cells. Moreover, IL-6 treatment exacerbated this difference, as indicated by the phosphorylation, but not the expression, of IL-6/JAK2/STAT3 signaling in PANC-1-TSA cells. Thus, over-activation of IL-6/JAK2/STAT3 signaling in PCC cells may be one explanation for TSA-induced resistance.

Expression levels of downstream target genes of IL-6/JAK2/STAT3 signaling in PCCs are upregulated by TSA. Given that TSA triggers the resistance in PCCs by inducing the activation of IL-6/JAK2/STAT3 signaling, we investigated whether TSA exerted similar effects on STAT3-downstream target genes. Thus, the mRNA expression levels of c-Myc, c-Src, HIF- $\alpha$, and CCND1 in PANC-1-TSA cells were quantified by the qRT-PCR. Enhanced levels of c-Myc and c-Src are required for the proliferation of PCCs, which are regulated by cyclin D1 (encoded by the CCND1 gene), a key molecule involved in regulating cell cycle progression (28-30). As shown in Fig. 2A, the mRNA expression levels of c-Myc, c-Src and CCND1 were markedly increased in PANC-1-TSA cells compared with those in PANC-1 cells $(\mathrm{P}<0.05)$, indicating that PANC-1-TSA cells may have a more proliferative activity than PANC-1 cells. In addition, overexpression of HIF- $\alpha$ mRNA was also observed in PANC-1-TSA cells (Fig. 2B). In response to a hypoxic tumor microenvironment, TSA-induced HIF- $\alpha$ expression plays an important role in differentiation, tumor growth and angiogenesis in pancreatic cancer (31). 
A

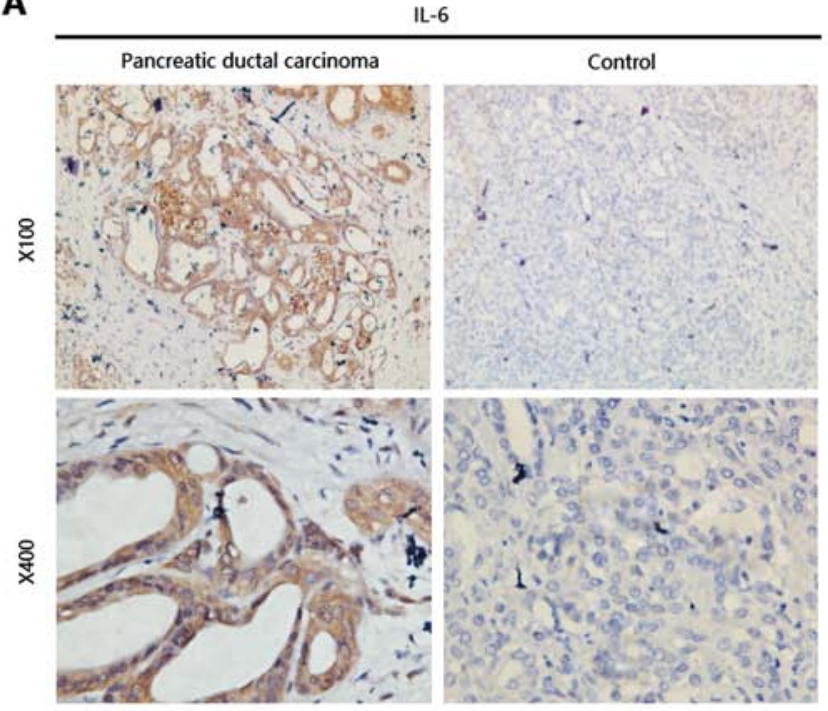

B

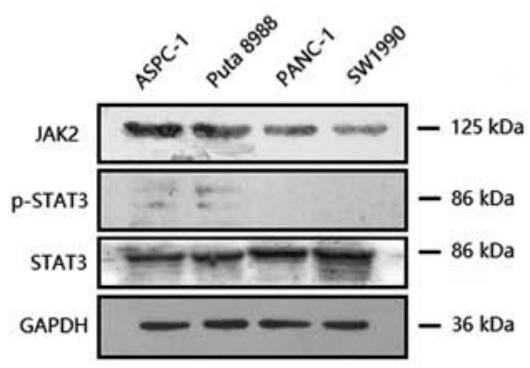

C

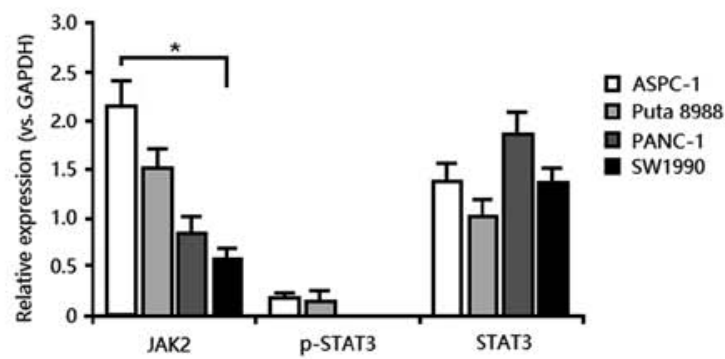

DMSO

口IL-6 $(100 \mathrm{ng} / \mathrm{ml})$

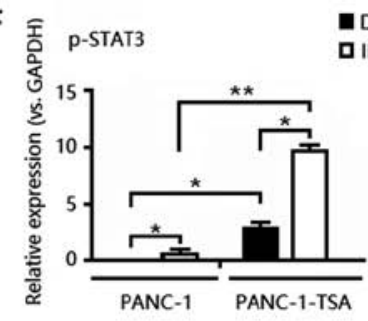

Figure 1. IL-6/JAK2/STAT3 signaling is activated in pancreatic cancer and exacerbated by TSA. (A) Enhanced expression of IL-6 in human pancreatic cancer tissues. Bar, $200 \mu \mathrm{m}$. (B) The expression of JAK2, STAT3 and phosphorylated STAT3 (p-STAT3) determined by western blotting was upregulated in different cell types, including PANC-1, ASPC-1, Puta 8988, and SW1990. (C) Relative expression levels of JAK2, STAT3 and p-STAT3 according to the results obtained with western blotting, ${ }^{*} \mathrm{P}<0.05$, SW1990 vs. ASPC-1. (D) The expression and phosphorylation of STAT3 in PANC-1 and PANC-1-TSA cells treated with DMSO or IL-6 (100 ng/ml) for $24 \mathrm{~h}$. (E) Relative expression levels of STAT3 according to the results obtained with western blotting. "P<0.05, vs. PANC-1 cells treated with DMSO. (F) The phosphorylation of STAT3 according to the results obtained with western blotting. ${ }^{*} \mathrm{P}<0.05$, vs. PANC-1 cells treated with DMSO; ${ }^{* *} \mathrm{P}<0.01$, PANC-1 cells with IL-6 (100 ng/ml) vs. PANC-1-TSA cells with IL-6 (100 ng/ml). PANC-1, non-resistant pancreatic cancer cells; PANC-1-TSA, TSA-resistant pancreatic cancer cells.

A

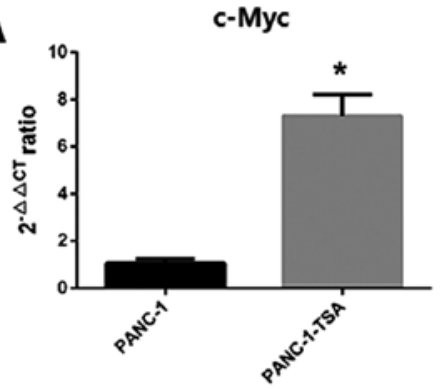

B

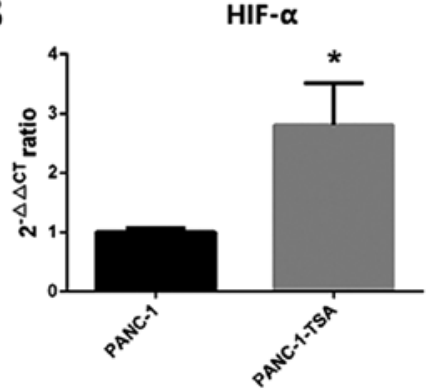

c-Src

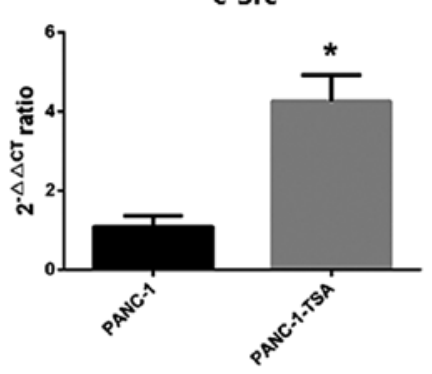

C

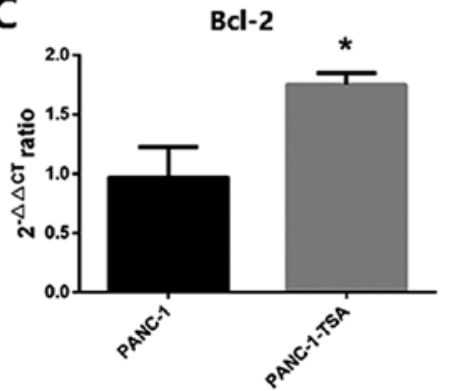

CCND1

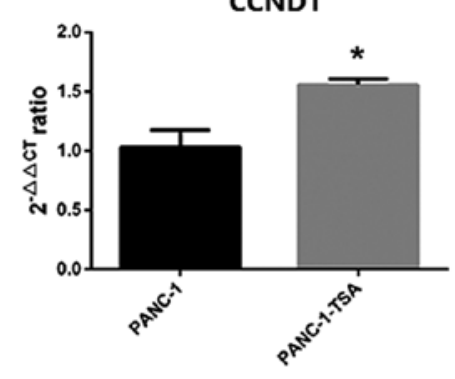

Bax

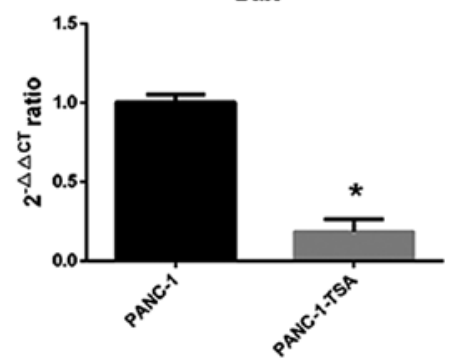

Figure 2. Expression levels of downstream target genes of IL-6/JAK2/STAT3 signaling in PCCs are upregulated by TSA. (A) Enhanced mRNA expression of c-Myc, c-Src and CCND1 in PANC-1-TSA cells compared with PANC-1 cells. "P<0.05, vs. PANC-1 cells. (B) Increased mRNA expression of HIF-1 $\alpha$ in PANC-1-TSA cells compared with PANC-1 cells. ${ }^{*} \mathrm{P}<0.05$, vs. PANC-1 cells. (C) Upregulated mRNA expression of Bcl-2 and downregulated mRNA expression of Bax in PANC-1-TSA cells compared with PANC-1 cells. " $\mathrm{P}<0.05$, vs. PANC-1 cells. PANC-1, non-resistant pancreatic cancer cells; PANC-1-TSA, TSA-resistant pancreatic cancer cells. 
A
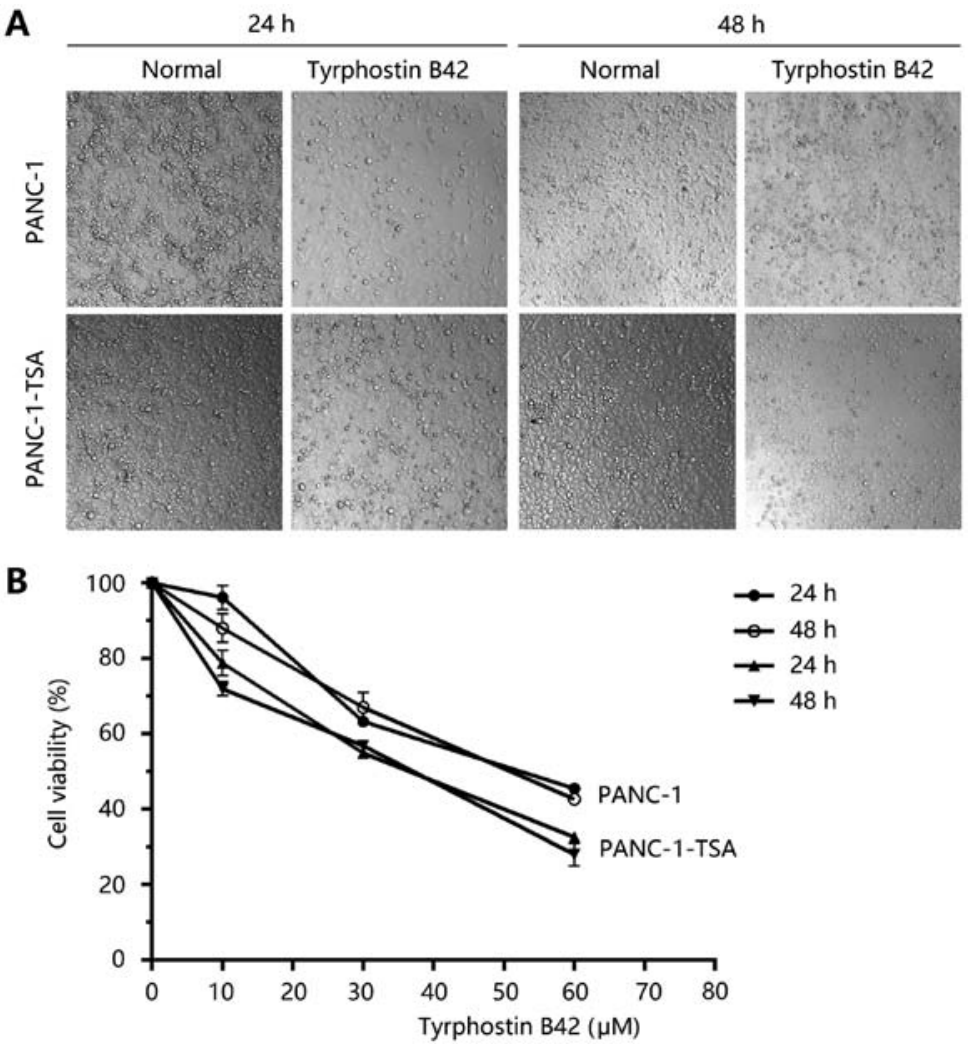

Figure 3. Tyrphostin B42 inhibits TSA-mediated over-proliferation of PCCs. (A) The effects of tyrphostin B42 (30 $\mu \mathrm{M})$ on the proliferation of PANC-1 and PANC-1-TSA cells at 24 and $48 \mathrm{~h}$ was observed under a microscope. After treatment with tyrphostin B $42(30 \mu \mathrm{M})$, the number of proliferated PCCs, especially for PANC-1-TSA cells, was significantly decreased. (B) A CCK-8 assay revealed the effects of tyrphostin B42 (0-60 $\mu \mathrm{M})$ on the proliferation of PANC-1 and PANC-1-TSA cells. PANC-1, non-resistant pancreatic cancer cells; PANC-1-TSA, TSA-resistant pancreatic cancer cells.

In addition to the induction of proliferation, TSA also exerted an inhibitory effect on the apoptosis of PCCs. As shown in Fig. 2C, the expression of Bcl-2 mRNA was upregulated in PANC-1-TSA cells whereas the expression of Bax was downregulated compared with that in PANC-1 cells $(\mathrm{P}<0.05)$, suggesting that TSA inhibited the apoptosis of PCCs through the mitochondrial pathway.

Tyrphostin B42 inhibits TSA-mediated over-proliferation of PCCs through IL-6/JAK2/STAT3 signaling. As aforementioned, PANC-1-TSA cells may possess more proliferative activity than PANC-1 cells. In this case whether tyrphostin B42 improves TSA-mediated resistance in PCCs through the inhibition of proliferation remains unknown. As shown in Fig. 3A, we determined that tyrphostin B42 $(30 \mu \mathrm{M})$ significantly decreased the number of proliferated PCCs, especially in PANC-1-TSA cells in a time-dependent manner. The results from the CCK-8 assay indicated that the viabilities of PANC-1 and PANC-1-TSA cells were decreased with the treatment of tyrphostin B42 (Fig. 3B, P<0.05). In addition, high-doses of tyrphostin $\mathrm{B} 42(\geq 30 \mu \mathrm{M})$ had a stronger inhibitory effect on the proliferation of PANC-1-TSA cells compared with PANC-1 cells (Fig. 3B, $\mathrm{P}<0.05$ ). These results revealed that tyrphostin B42 inhibited the over-proliferative activity of PCCs and improved TSA-mediated resistance.

After TSA treatment, IL-6/JAK2/STAT3 signaling was activated and then induced the over-proliferation of PCCs. In the present experiment, in PANC-1-TSA cells, tyrphostin B42 reduced the expression of STAT3 and inhibited the phos-

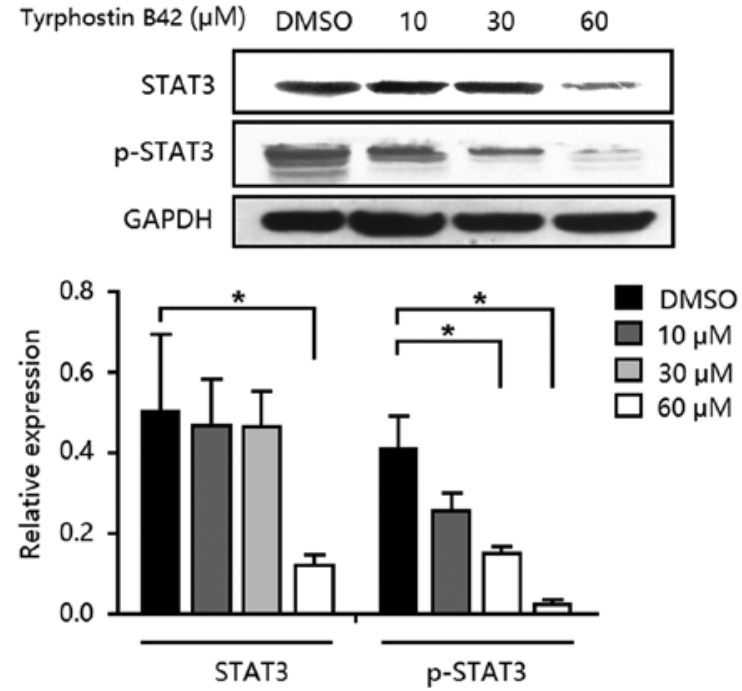

Figure 4. Effects of tyrphostin B42 on the IL-6/JAK2/STAT3 signaling in PANC-1-TSA cells. Tyrphostin B42 $(0-60 \mu \mathrm{M})$ markedly inhibited TSA-mediated overexpression and phosphorylation of STAT3 in PANC-1 cells for $24 \mathrm{~h}$ in a dose-dependent manner as determined by western blotting. ${ }^{*} \mathrm{P}<0.05$, vs. PANC-1-TSA cells treated with DMSO.

phorylation of p-STAT3 (Fig. 4). Moreover, high-doses of tyrphostin $\mathrm{B} 42(\geq 30 \mu \mathrm{M})$ exerted stronger inhibitory effects on IL-6/JAK2/STAT3 signaling. Consequently, tyrphostin B42-mediated inhibition of PCC proliferation may be induced through IL-6/JAK2/STAT3 signaling. 

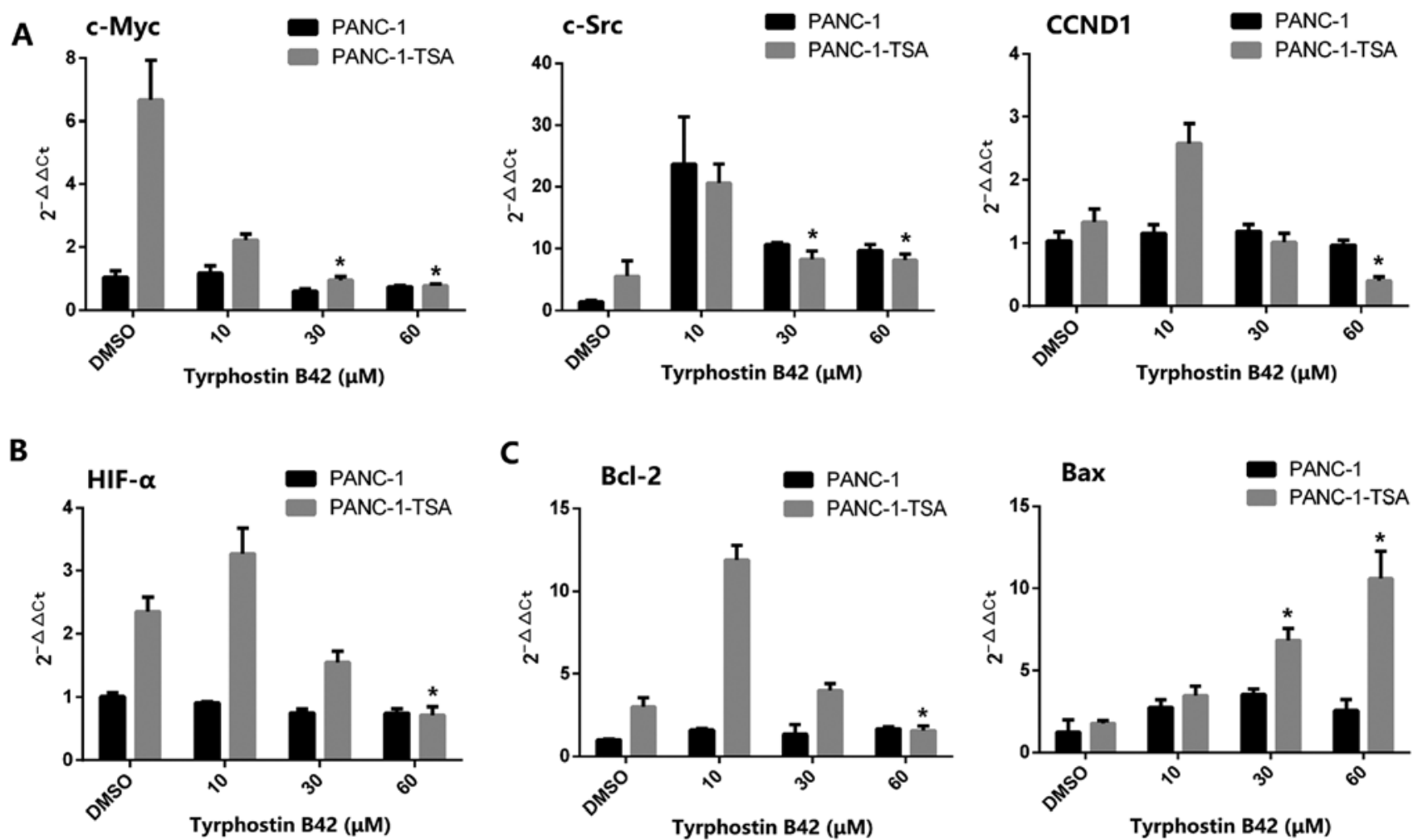

Figure 5. Tyrphostin B42 inhibits the expression of downstream target genes of IL-6/JAK2/STAT3 signaling. (A) High-doses of tyrphostin B42 ( $\geq 30 \mu \mathrm{M}$ ) significantly reduced the mRNA expression of c-Myc, c-Src and CCND1 in PANC-1-TSA cells for $24 \mathrm{~h}$. "P<0.05, vs. PANC-1-TSA cells treated with a low-dose of tyrphostin B42 (10 $\mu \mathrm{M})$. (B) High-doses of tyrphostin B42 $(\geq 30 \mu \mathrm{M})$ decreased the mRNA expression of HIF-1 $\alpha$ in PANC-1-TSA cells. "P<0.05, vs. PANC-1-TSA cells treated with a low-dose of tyrphostin B42 $(10 \mu \mathrm{M})$. (C) High-doses of tyrphostin B42 $(\geq 30 \mu \mathrm{M})$ downregulated the mRNA expression of $\mathrm{Bcl}-2$ and upregulated the expression of Bax in PANC-1-TSA cells. ${ }^{*} \mathrm{P}<0.05$, vs. PANC-1-TSA cells treated with a low-dose of tyrphostin B42 (10 $\left.\mu \mathrm{M}\right)$. PANC-1, non-resistant pancreatic cancer cells; PANC-1-TSA, TSA-resistant pancreatic cancer cells.

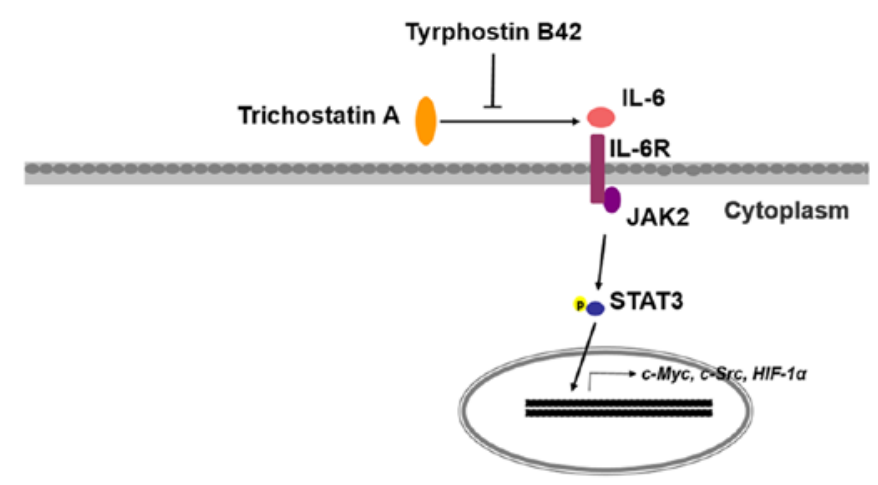

Figure 6. A brief diagram displaying the interactions involved in the IL-6/JAK2/STAT3 pathway with interventions including HDAC inhibitor TSA and antagonist Tyrphostin B42.

Tyrphostin $B 42$ inhibits the expression of downstream target genes of IL-6/JAK2/STAT3 signaling. In PANC-1 cells, tyrphostin B42 $(0-60 \mu \mathrm{M})$ did not downregulate the expression of downstream target genes of IL-6/JAK2/STAT3 signaling, including c-Myc, c-Src and CCND1 (Fig. 5A). Similarly, in PANC-1-TSA cells, a low-dose $(<30 \mu \mathrm{M})$ of tyrphostin B42 did not inhibit but promote the expression of these target genes. However, high-doses $(\geq 30 \mu \mathrm{M})$ of tyrphostin $\mathrm{B} 42$ downregulated the mRNA expression of c-Myc, c-Src and CCND1. In addition, high-doses of tyrphostin B42 also reduced low-dose-mediated overexpression of HIF- $\alpha$ mRNA in PANC-1-TSA cells (Fig. 5B). Furthermore, decreased expression of $\mathrm{Bcl}-2$ and increased expression of $\mathrm{Bax}$ in PANC-1-TSA cells were inhibited with tyrphostin B42 treatment at low doses $(<30 \mu \mathrm{M})$ (Fig. 5C). These results indicated that tyrphostin $\mathrm{B} 42$ at higher doses $(\geq 30 \mu \mathrm{M})$ exerted an inhibitory effect on the expression of downstream target genes of IL-6/JAK2/STAT3 signaling, leading to the inhibition of proliferation, the induction of apoptosis, and the reduction of TSA-mediated resistance in PCCs.

\section{Discusssion}

Pancreatic cancer is considered to be one of the most lethal solid tumors with early metastasis and high resistance to chemotherapy (19). Multiple biochemical and molecular alterations, including genetic alterations, epigenetic changes, redundancies and crosstalk of cell signaling pathways, contribute to the development and drug-resistance of pancreatic cancer (28). In addition, abnormal activation of some pathways may be involved in resistance to pancreatic cancer chemotherapy. Accumulating studies provide evidence that the IL6/JAK2/STAT3 signaling pathway plays an important role in drug resistance and is highly expressed in different types of drug-resistant cancers, including pancreatic cancer. IL-6 is a pleiotropic cytokine produced by a variety of cell types including macrophages, fibroblasts and cancer cells. IL-6 binds to membrane receptors (e.g., IL-6R) and then activates non-receptor tyrosine kinases, including JAK2. 
These phosphotyrosine residues serve as a docking site for the recruitment of STAT3 proteins, which act as cellular mediators of IL-6 (39). STAT3 is an oncogene that is activated by $\mathrm{p}-\mathrm{STAT} 3$ in response to extracellular signals and JAK2 pathway activation (30). Once the tyrosine is phosphorylated, two STAT3 monomers form dimers through reciprocal phosphotyrosine-SH2 interactions, translocate to the nucleus, where they bind to STAT3-specific DNA-response elements of target genes, and induce gene transcription (31). Thus, IL-6 induces the activation of its downstream cascade the JAK2/STAT3 signaling pathway, resulting in tumorigenesis by regulating cell cycle progression, angiogenesis and tumor cell evasion of the immune system (32-34). Evidence reveals an important role of IL-6/JAK2/STAT3 signaling in the development of pancreatic cancer (31).

It has been reported that PCCs were resistant to HDAC inhibitor trichostatin A (TSA) (8). Aberrant activation of Wnt/ $\beta$-catenin signaling contributes to TSA resistance through epithelial-mesenchymal transition. In the present study, we initially observed increased IL-6 expression in human pancreatic tissues and identified the crucial role of JAK2/STAT3 signaling in TSA resistance in PCCs. The elevated levels of IL-6 were positively associated with lymph node metastasis, tumor differentiation and vascular infiltration of pancreatic cancer. TSA-resistant cells exhibited significantly upregulated expression and phosphorylation of STAT3 along with enhanced expression of c-Myc, c-Src, HIF- $\alpha$, and CCND1 as compared to TSA-nonresistant cells. Moreover, in aggressive malignant pancreatic cancer cell lines, the significantly elevated expression of IL- 6 predicted a more aggressive cell type and a poorer clinical outcome. On the basis of these data, JAK2 could be considered as a potential target for chemotherapy-resistant pancreatic carcinoma. Thus, targeted inhibition of the over-activation of IL-6/JAK2/STAT3 signaling can provide a strategy for the treatment of TSA resistance.

Tyrphostin B42 (AG490) is an inhibitor of epidermal growth factor receptor (EGFR) by competing to binding sites with receptor tyrosine kinases (RTKs) (35). Tyrphostin B42 has been reported to have an inhibitory effect on the proliferation of many tumor cells through IL-6/JAK2/STAT3 signaling (22-24). A previous study revealed that in vitro AG-490 (60-100 $\mu \mathrm{M})$ blocked the constitutive activation of Stat $3^{\text {sm }}$, and inhibited spontaneous as well as interleukin 2-induced growth of mycosis fungoides tumor cells (36). In vivo, combined therapy with AG-490 and IL-12 induced greater antitumor effects than either agent alone in a murine myeloma tumor model (37). In the present study, we found that tyrphostin B42 (30-60 $\mu \mathrm{M})$ inhibited the proliferation of PCCs in a dose-dependent manner. The inactivation of IL-6/JAK2/STAT3 signaling was responsible for tyrphostin B42-mediated inhibition of proliferation of PCCs. Inactivated IL-6/JAK2/STAT3 signaling resulted in the imbalance of proliferation- and apoptosis-associated gene expression. Furthermore, tyrphostin B42 also inhibited the expression of c-Src mRNA in non-resistant PCCs, indicating that tyrphostin B42 not only attenuates TSA-mediated resistance, but also has a therapeutic potential for pancreatic cancer.

However, an evident limitation in the present study is that it did not build TSA-resistant tumor animal models to evaluate the protective effects of tyrphostin B42 in vivo. In addition, tyrphostin B42 treatment at low concentrations (less than
$10 \mu \mathrm{M})$ did not exert an inhibitory effect on the expression of downstream target genes of IL-6/JAK2/STAT3 signaling, revealing that other mechanisms appear to be involved in the regulation of proliferation and apoptosis of TSA-mediated resistance in PCCs, and thus it is important for tyrphostin B42 to have an appropriate dose/concentration used in vivo.

In conclusion, our in vitro experiments indicated that aberrant activation of IL-6/JAK2/STAT3 signaling was likely one of the main mechanisms triggering the resistance to TSA, leading to over-proliferation and inhibition of apoptosis of PCCs (Fig. 6). Tyrphostin B42 at certain concentrations effectively attenuates TSA-mediated resistance in PCCs by antagonizing IL-6/JAK2/STAT3 signaling. The present study helps to better understand the TSA-resistance mechanism in pancreatic cancer, and provide a theoretical basis for the screening of antitumor drugs.

\section{Acknowledgements}

This study was supported by the Natural Science Foundation of Zhejiang province, China (LY17H050005), the National Natural Science Foundation of China (nos. 81570583 and 81572087), and the Wenzhou Municipal Science and Technology Plan Project (Y20150037).

\section{Competing interests}

The authors declare that they have no competing interests.

\section{References}

1. Castellanos JA, Merchant NB and Nagathihalli NS: Emerging targets in pancreatic cancer: Epithelial-mesenchymal transition and cancer stem cells. Onco Targets Ther 6: 1261-1267, 2013.

2. Siegel R, Naishadham D and Jemal A: Cancer statistics, 2013. CA Cancer J Clin 63: 11-30, 2013.

3. Ouaïssi M, Cabral S, Tavares J, da Silva AC, Mathieu Daude F, Mas E, Bernard J, Sastre B, Lombardo D and Ouaissi A: Histone deacetylase (HDAC) encoding gene expression in pancreatic cancer cell lines and cell sensitivity to HDAC inhibitors. Cancer Biol Ther 7: 523-531, 2008.

4. Donadelli M, Costanzo C, Beghelli S, Scupoli MT, Dandrea M, Bonora A, Piacentini P, Budillon A, Caraglia M, Scarpa A and Palmieri M: Synergistic inhibition of pancreatic adenocarcinoma cell growth by trichostatin A and gemcitabine. Biochim Biophys Acta 1773: 1095-1106, 2007.

5. Zhu W, Li J, Wu S, Li S, Le L, Su X, Qiu P, Hu H and Yan G: Triptolide cooperates with Cisplatin to induce apoptosis in gemcitabine-resistant pancreatic cancer. Pancreas 41: 1029-1038, 2012.

6. Kim DH, Kim M and Kwon HJ: Histone deacetylase in carcinogenesis and its inhibitors as anti-cancer agents. J Biochem Mol Biol 36: 110-119, 2003.

7. Zhang X, Jiang SJ, Shang B and Jiang HJ: Effects of histone deacetylase inhibitor trichostatin A combined with cisplatin on apoptosis of A549 cell line. Thorac Cancer 6: 202-208, 2015.

8. Wang B, Zou Q, Sun M, Chen J, Wang T, Bai Y, Chen Z, Chen B and Zhou M: Reversion of trichostatin A resistance via inhibition of the Wnt signaling pathway in human pancreatic cancer cells. Oncol Rep 32: 2015-2022, 2014.

9. Chen Z, Yang Y, Liu B, Wang B, Sun M, Zhang L, Chen B, You $\mathrm{H}$ and Zhou M: Promotion of metastasis-associated gene expression in survived PANC-1 cells following Trichostatin a treatment. Anticancer Agents Med Chem 15: 1317-1325, 2015.

10. Taniguchi K and Karin M: IL-6 and related cytokines as the critical lynchpins between inflammation and cancer. Semin Immunol 26: 54-74, 2014.

11. Okitsu K, Kanda T, Imazeki F, Yonemitsu Y, Ray RB, Chang C and Yokosuka O: Involvement of interleukin- 6 and androgen receptor signaling in pancreatic cancer. Genes Cancer 1: 859-867, 2010. 
12. Bharadwaj U, Marin-Muller C, Li M, Chen C and Yao Q Mesothelin overexpression promotes autocrine IL-6/sIL-6R trans-signaling to stimulate pancreatic cancer cell proliferation. Carcinogenesis 32: 1013-1024, 2011.

13. Vera J, Rateitschak K, Lange F, Kossow C, Wolkenhauer O and Jaster R: Systems biology of JAK-STAT signalling in human malignancies. Prog Biophys Mol Biol 106: 426-434, 2011.

14. Quintás-Cardama A and Verstovsek S: Molecular pathways: Jak/STAT pathway: Mutations, inhibitors, and resistance. Clin Cancer Res 19: 1933-1940, 2013.

15. Thoennissen NH, Iwanski GB, Doan NB, Okamoto R, Lin P, Abbassi S, Song JH, Yin D, Toh M, Xie WD, et al: Cucurbitacin B induces apoptosis by inhibition of the JAK/STAT pathway and potentiates antiproliferative effects of gemcitabine on pancreatic cancer cells. Cancer Res 69: 5876-8584, 2009.

16. Gozgit JM, Bebernitz G, Patil P, Ye M, Parmentier J, Wu J, $\mathrm{Su} N$, Wang T, Ioannidis S, Davies A, et al: Effects of the JAK2 inhibitor, AZ960, on Pim/BAD/BCL-xL survival signaling in the human JAK2 V617F cell line SET-2. J Biol Chem 283: 32334-32343, 2008.

17. Bai J, Sui J, Demiriian A, Vollmer CM Jr, Marasco W and Callery MP: Predominant Bcl-XL knockdown disables antiapoptotic mechanisms: tumor necrosis factor-related apoptosis-inducing ligand-based triple chemotherapy overcomes chemoresistance in pancreatic cancer cells in vitro. Cancer Res 65: 2344-2352, 2005.

18. Hodge DR, Hurt EM and Farrar WL: The role of IL-6 and STAT3 in inflammation and cancer. Eur J Cancer 41: 2502-2512, 2005.

19. Wang R, Cheng L, Xia J, Wang Z, Wu Q and Wang Z: Gemcitabine resistance is associated with epithelial-mesenchymal transition and induction of HIF-1 $\alpha$ in pancreatic cancer cells. Curr Cancer Drug Targets 14: 407-417, 2014.

20. Chen H, Guan YL, Yuan G, Zhang Q and Jing N: A perylene derivative regulates HIF-1 alpha and Stat 3 signaling pathways Bioorg Med Chem 22: 1496-1505, 2014

21. Qin A, Yu Q, Gao Y, Tan J, Huang H, Qiao Z and Qian W: Inhibition of STAT3/cyclin D1 pathway promotes chemotherapeutic sensitivity of colorectal caner. Biochem Biophys Res Commun 457: 681-687, 2015.

22. Soto-Cruz I, Rangel-Corona R, Valle-Mendiola A Moreno-Morales X, Santiago-Pérez R, Weiss-Steider B and Cáceres-Cortés JR: The tyrphostin B42 inhibits cell proliferation and HER-2 autophosphorylation in cervical carcinoma cell lines. Cancer Invest 26: 136-144, 2008

23. Xu Y, Zhang J, Wu J, Zhong $\mathrm{S}$ and $\mathrm{Li} \mathrm{H}$ : Inhibition of JAK2 Reverses paclitaxel resistance in human ovarian cancer cells. Int J Gynecol Cancer 25: 1557-1564, 2015.

24. Chen B, Liu Y, Zhang Y, Li J, Cheng K and Cheng L: IL-21 is positively associated with intervertebral disc degeneration by interaction with TNF- $\alpha$ through the JAK-STAT signaling pathway. Inflammation 40: 612-622, 2017.
25. American Joint Committee on Cancer (AJCC) TNM staging system (2013) American Cancer Society. http://www.cancer.org/ cancer/pancreaticcancer/detailedguide/pancreatic-cancer-staging. Accessed April 5, 2016.

26. Detre S, Saclani Jotti G and Dowsett MA: A 'quickscore' method for immunohistochemical semiquantitation: Validation for oestrogen receptor in breast carcinomas. J Clin Pathol 48: 876-878, 1995.

27. Livak KJ and Schmittgen TD: Analysis of relative gene expression data using real-time quantitative PCR and the 2(-Delta Delta C(T)) method. Methods 25: 402-408, 2001.

28. Silvestris N, Gnoni A, Brunetti AE, Vincenti L, Santini D, Tonini G, Merchionne F, Maiello E, Lorusso V, Nardulli P, et al: Target therapies in pancreatic carcinoma. Curr Med Chem 21: 948-965, 2014

29. Roxburgh CS and McMillan DC: Therapeutics targeting innate immune/inflammatory responses through the interleukin-6/JAK/STAT signal transduction pathway in patients with cancer. Transl Res 167: 61-66, 2016.

30. Kim BH, Yi EH and Ye SK: Signal transducer and activator of transcription 3 as a therapeutic target for cancer and the tumor microenvironment. Arch Pharm Res 39: 1085-1099, 2016.

31. Bowman T, Garcia R, Turkson J and Jove R: STATs in oncogenesis. Oncogene 19: 2474-2488, 2000.

32. Pop VV, Seicean A, Lupan I, Samasca G and Burz CC: IL-6 roles-Molecular pathway and clinical implication in pancreatic cancer-A systemic review. Immunol Lett 181: 45-50, 2017.

33. Miyamoto Y, Hosotani R, Doi R, Wada M, Ida J, Tsuji S, Kawaguchi M, Nakajima S, Kobayashi H, Masui T, et al: Interleukin- 6 inhibits radiation induced apoptosis in pancreatic cancer cells. Anticancer Res 21: 2449-2456, 2001

34. Fofaria NM and Srivastava SK: STAT3 induces anoikis resistance, promotes cell invasion and metastatic potential in pancreatic cancer cells. Carcinogenesis 36: 142-150, 2015.

35. Caceres-Cortes JR: A potent anti-carcinoma and anti-acute myeloblastic leukemia agent, AG490. Anticancer Agents Med Chem 8: 717-722, 2008.

36. Nielsen M, Kaltoft K, Nordahl M, Röpke C, Geisler C, Mustelin T, Dobson P, Svejgaard A and Odum N: Constitutive activation of a slowly migrating isoform of Stat 3 in mycosis fungoides: Tyrphostin AG490 inhibits Stat3 activation and growth of mycosis fungoides tumor cell lines. Proc Natl Acad Sci USA 94: 6764-6769, 1997.

37. Burdelya L, Catlett-Falcone R, Levitzki A, Cheng F, Mora LB, Sotomayor E, Coppola D, Sun J, Sebti S, Dalton WS, et al: Combination therapy with AG-490 and interleukin 12 achieves greater antitumor effects than either agent alone. Mol Cancer Ther 1: 893-899, 2002. 